Bozena Swiatek, Institute of Mathematics, Łódź University, ul. Stefana Banacha 22, 90-238 Łódź, Poland.

\title{
EXTENDING DARBOUX FUNCTIONS WITH FINITE VARIATION
}

\begin{abstract}
In this paper we show that a Darboux function with finite variation, which is defined on closed, convex and boundary subset of $\mathbb{R}^{2}$, can be extended to a Darboux function with finite variation, which is defined on $\mathbb{R}^{2}$. Moreover, the set of all points of continuity and the set of all points of quasi-continuity for the first function are equal to the corresponding sets for the extension of this function.
\end{abstract}

In many papers concerning real functions of a real variable, the problem of the "variation of the function" plays an important role. Consequently many mathematicians dealt with the variation of functions defined on more abstract spaces (see for example, [7]). It is known (see [3]) that if $f:[0,1] \rightarrow \mathbb{R}$ is a continuous function and $N_{f}$ denotes a Banach indicatrix of the function $f$, then $N_{f}$ is a measurable function and the variation $\bigvee_{a}^{b}(f)$ of the function $f$ on the interval $[\mathrm{a}, \mathrm{b}]$ equals $\int_{-\infty}^{+\infty} N_{f}(y) d y$. (T. Salat in [6] proved that this holds also for Darboux functions.) This allows one to generalize the notion of variation for functions which are defined and take their values in more general spaces. Let us first define Banach indicatrix.

Definition 1. We define the Banach indicatrix of a function $f: E \rightarrow \mathbb{R}^{2}$ $\left(E \subset \mathbb{R}^{2}\right)$ to be the function $N_{f}: \mathbb{R}^{2} \rightarrow \mathbb{R}$ such that $N_{f}$ equals the number of points of the set $f^{-1}(p)$ when this set is finite, and equals $+\infty$ when it is infinite.

Definition 2. Let $f: E \rightarrow \mathbb{R}^{2}\left(E \subset \mathbb{R}^{2}\right)$ be a function whose Banach indicatrix $N_{f}$ is measurable. Then $f$ is called a function with finite variation if $\int_{\mathbb{R}^{2}} N_{f}(p) d p<+\infty$.

Mathematical Reviews subject classification: 26B30

Received by the editors November 7, 1994 
In [3] properties of Darboux functions with finite variation, mapping $\mathbb{R}^{2}$ into $\mathbb{R}^{2}$, were considered for the first time. These considerations concerned, among others things, the existence of mappings which are not continuous. In [4] A. Rychlewicz showed that, in the topological sense, there are a lot of such functions. He also considered the problem of extending Darboux functions with finite variation. He proved the following assertion.

Theorem 1. Let $\mathcal{K}$ be a closed convex subset of $\mathbb{R}^{2}$ which is not a boundary set. Let $f: \mathcal{K} \rightarrow \mathbb{R}^{2}$ be a Darboux function with finite variation. Then, for each connected and stratiformly locally connected (with respect to $\mathcal{K}$ ) subspace $\mathcal{X}$ of $\mathbb{R}^{2}$, containing $\mathcal{K}$, there exists a Darboux function $f^{\star}: \mathcal{X} \rightarrow \mathbb{R}^{2}$ with finite variation which is an extension of $f$ on $\mathcal{X}$ such that

$$
Q_{f}=Q_{f^{\star}} \subset \tilde{Q}_{f^{\star}}(\mathcal{K}) .
$$

In this paper we shall show that we need not assume that $\mathcal{K}$ has nonempty interior. We follow the notation used in, e.g., [1] or [3]. But we take a "stronger" version of the definition of a Darboux function.

Definition 3. A function $f: X \rightarrow Y$ (X, $Y$ - topological spaces) is called a Darboux function if $f(C)$ is a connected set for each connected set $C \subset X$.

(Since the initial functions will be defined on convex boundary sets, our version of the theorem is stronger than those with the definition from works [3] and [4]). Let $\Phi$ denote the set $\left\{(x, y) \in \mathbb{R}^{2}: x \in \mathbb{Q} \vee y \in \mathbb{Q}\right\}$ where $\mathbb{Q}$ denotes the set of rational numbers. Let $\bar{K}(a, \varepsilon)$ stand for the closed ball with center a and radius $\varepsilon$, i.e. $\bar{K}(a, \varepsilon)=\{z: \rho(a, z) \leq \varepsilon\}$ where $\rho$ denotes the metric on the plane $\mathbb{R}^{2}$.

Definition 4. We say that a function $f: X \rightarrow Y(X, Y-$ metric spaces $)$ is quasi-continuous at $x_{0}$ if for each neighborhood $U$ of the point $x_{0}$ and for each neighborhood $V$ of the point $g\left(x_{0}\right)$, there exists a non-empty open set $G \subset U$ such that $f(G) \subset V$.

If $f: X \rightarrow \mathbb{R}^{2}$ (where $X=\mathbb{R}$ or $X=\mathbb{R}^{2}$ ), then by $Q_{f}\left(C_{f}\right)$ we denote the set of all points of quasi-continuity (continuity) of the function $f$. If $\star$ is an equivalence relation then the abstract class of this relation determined by an element $\alpha$ is denoted by $[\alpha]_{\star}$. As in paper [3] we adopt the following definitions.

Definition 5. Let $X$ be a set. Let $(Y, \rho)$ be a metric space, $A \subset X$ and $f: A \rightarrow Y$. A function $f^{\star}: X \rightarrow Y$ is called an $\varepsilon$-extension of the function $f(\varepsilon \geq 0)$ if $f^{\star}$ is an extension of $f$ and for each $\alpha \in f^{\star}(X)$, there exists $\beta \in f(A)$ such that $\rho(\alpha, \beta) \leq \varepsilon$. 
Definition 6. We say that a non-empty closed set $K$ cuts a topological space $X$ (between non-empty sets $A$ and $B$ ) if $X \backslash K=U \cup V$ where $U$ and $V$ are non-empty open sets such that $U \cap V=\emptyset$ and $A \subset U, B \subset V$.

If $A$ and $B$ are subsets of some topological space $X$, then the symbol $\operatorname{int}_{A}(B)$ denotes the interior of the set $B$ in $A$ being a subspace of $X$. If $A=X$, we simply write $\operatorname{int}(B)$. A projection onto the space $X$ is denoted by $\operatorname{proj}_{X}$.

First, we shall prove some lemmas.

Lemma 1. Let $A \subset \mathbb{R}^{2}$. If $U \subset \mathbb{R}^{2}$ is an open set such that $U \cup A$ is connected, then $(U \cap \Phi) \cup A$ is a connected set.

Proof.Suppose that $(U \cap \Phi) \cup A$ is not connected. Then $(U \cap \Phi) \cup A=W \cup T$ where $\mathrm{W}$ and $\mathrm{T}$ are non-empty and separated sets, i.e.

$$
U \backslash \Phi \subset U \subset \operatorname{cl}(U \cap \Phi) \subset \operatorname{cl}(W) \cup \operatorname{cl}(T)
$$

and

$$
T \cup W \cup(U \backslash \Phi)=U \cup A .
$$

Therefore, by (1) and (2),

$$
U \cup A=(T \cup((U \backslash \Phi) \cap \operatorname{cl}(T))) \cup(W \cup((U \backslash \Phi) \cap \operatorname{cl}(W))) .
$$

Moreover, note that

$$
T \cup((U \backslash \Phi) \cap c l(T)) \neq \emptyset \quad \text { and } \quad W \cup((U \backslash \Phi) \cap c l(W)) \neq \emptyset .
$$

Now, it will be shown that

$$
T \cup((U \backslash \Phi) \cap \operatorname{cl}(T)) \text { and } W \cup((U \backslash \Phi) \cap \operatorname{cl}(W)) \text { are separated. }
$$

Indeed, we have

$$
\begin{aligned}
& \operatorname{cl}(T \cup((U \backslash \Phi) \cap \operatorname{cl}(T))) \cap(W \cup((U \backslash \Phi) \cap \operatorname{cl}(W))) \\
= & (\operatorname{cl}(T) \cap \operatorname{cl}(W) \cap(U \backslash \Phi)) \cup(\operatorname{cl}((U \backslash \Phi) \cap \operatorname{cl}(T)) \cap \operatorname{cl}(W) \cap(U \backslash \Phi)) \\
= & \operatorname{cl}(T) \cap \operatorname{cl}(W) \cap(U \backslash \Phi) .
\end{aligned}
$$

Suppose that there exists $x_{0} \in \operatorname{cl}(T) \cap \operatorname{cl}(W) \cap(U \backslash \Phi)$. Then there exists $r>0$ such that $K\left(x_{0}, r\right) \subset U$. Then

$$
K\left(x_{0}, r\right) \cap T \neq \emptyset \quad \text { and } \quad K\left(x_{0}, r\right) \cap W \neq \emptyset .
$$


Also, $K\left(x_{0}, r\right) \cap \Phi \subset U \cap \Phi \subset W \cup T$. Evidently, $K\left(x_{0}, r\right) \cap \Phi$ is a connected set, so $K\left(x_{0}, r\right) \cap \Phi \subset W$ or $K\left(x_{0}, r\right) \cap \Phi \subset T$. If $K\left(x_{0}, r\right) \cap \Phi \subset W$, then, by (5), $\operatorname{cl}(W) \cap T \supset K\left(x_{0}, r\right) \cap T \neq \emptyset$. If $K\left(x_{0}, r\right) \cap \Phi \subset T$, then, by (5), $\operatorname{cl}(T) \cap W \supset K\left(x_{0}, r\right) \cap W \neq \emptyset$, which contradicts the fact that $\mathrm{W}$ and $\mathrm{T}$ are separated sets. We have thus proved that $\operatorname{cl}(T) \cap \operatorname{cl}(W) \cap(U \backslash \Phi)=\emptyset$. Hence $\operatorname{cl}(T \cup((U \backslash \Phi) \cap \operatorname{cl}(T))) \cap(W \cup((U \backslash \Phi) \cap \operatorname{cl}(W))=\emptyset$. In a similar way we can prove that

$$
(T \cup((U \backslash \Phi) \cap \operatorname{cl}(T))) \cap \operatorname{cl}(W \cup((U \backslash \Phi) \cap \operatorname{cl}(W)))=\emptyset,
$$

which gives (4). By (3) and (4), this contradicts the connectedness of the set $U \cup A$. Thus we have proved that $(U \cap \Phi) \cup A$ is connected.

Corollary 1. If $U \subset \mathbb{R}^{2}$ is an open connected set, then $U \cap \Phi$ is connected

Lemma 2. Let $F \subset \mathbb{R}$ be a closed convex set. Let $f: F \rightarrow \mathbb{R}^{2}$ be a Darboux function with finite variation. Then, for any $\varepsilon>0$, there exists a Darboux function $g: \mathbb{R} \rightarrow \mathbb{R}^{2}$ with finite variation which is an $\varepsilon$ extension of $f$ such that $Q_{g}=Q_{f}$ and $C_{g}=C_{f}$.

Proof.Obviously, $F$ is a line, a closed half-line, a non-degenerate closed segment, or a point. If $F=\mathbb{R}$, we obviously put $g=f$. Hence, in the remainder of the proof, we may assume that $F \neq \mathbb{R}$. First, we shall consider the case when $F$ is a closed half-line. Let $p$ denote its endpoint. Assume that $F=(-\infty, p]$ (if $F=[p,+\infty)$, the proof is analogous). Let $\varepsilon>0$. Consider two cases: $1^{0} p \notin Q_{f}$.

Then in $\mathbb{R} \backslash F$ we define the equivalence relation $\star$ by

$$
x \star y \Longleftrightarrow x-y \in \mathbb{Q} \text {. }
$$

Let $\mathcal{D}$ denote the set of abstract classes of the relation $\star$. Then there exists a bijection $h: \mathcal{D} \stackrel{\text { onto }}{\longmapsto} K(f(p), \varepsilon) \cap \Phi$. We define $g: \mathbb{R} \rightarrow \mathbb{R}^{2}$ by

$$
g(x)= \begin{cases}f(x), & \text { if } x \in F, \\ h\left([x]_{\star}\right), & \text { if } x \in \mathbb{R} \backslash F .\end{cases}
$$

Evidently,

$$
g \text { is an } \varepsilon-\text { extension of } f \text {. }
$$

Now, we shall show that

$$
g \text { is a Darboux function. }
$$

Let $C \subset \mathbb{R}$ be a connected set. Consider the following cases. 
1) $C \subset F$; then $\mathrm{g}(\mathrm{C})=\mathrm{f}(\mathrm{C})$ is connected.

2) $C \subset \mathbb{R} \backslash F$.

2a) $\mathrm{C}$ is a point, so is $\mathrm{g}(\mathrm{C})$.

2b) $\mathrm{C}$ is a non-degenerate segment or a half-line.

Then, for any abstract class $[x]_{\star} \in \mathcal{D},[x]_{\star} \cap C \neq \emptyset$. Hence $g(C)=K(f(p), \varepsilon) \cap$ $\Phi$ is a connected set.

3) $C \cap F \neq \emptyset$ and $C \backslash F \neq \emptyset$.

Note that, in this case,

$g(C \cap F)$ and $g(C \backslash F)$ are non - empty connected and non - separated sets.

Since $g(C)=g(C \cap F) \cup g(C \backslash F)$, we conclude from (8) that $g(C)$ is connected. This proves $(7)$.

Now, we shall show that $Q_{g}=Q_{f}$. Obviously, Therefore it sufficient to prove that $Q_{g} \subset Q_{f}$. For the purpose, we shall first show that

$$
p \notin Q_{g} .
$$

Indeed, since $p \notin Q_{f}$, there exist $0<\varepsilon_{0}<\varepsilon$ and $\delta_{0}>0$ such that

for any non-emptyset $U \subset\left(p-\delta_{0}, p\right]$ open in $F f(U) \not \subset K\left(f(p), \varepsilon_{0}\right)$.

To prove $p \notin Q_{g}$,it is sufficient to show that

for any non-empty set $V \subset\left(p-\delta_{0}, p+\delta_{0}\right), g(V) \not \subset K(f(p), \varepsilon)$.

Let $V \subset\left(p-\delta_{0}, p+\delta_{0}\right)$ be a non-empty open set. If $V \cap\left(p-\delta_{0}, p\right) \neq \emptyset$, then $g\left(V \cap\left(p-\delta_{0}, p\right)\right) \not \subset K\left(f(p), \varepsilon_{0}\right)$ by (10), and so, $g(V) \not \subset K\left(f(p), \varepsilon_{0}\right)$. If $V \cap\left(p-\delta_{0}, p\right)=\emptyset$, then $g(V)=K(f(p), \varepsilon) \cap \Phi$. If $g(V) \subset K\left(f(p), \varepsilon_{0}\right)$, then $\bar{K}(f(p), \varepsilon) \subset \bar{K}\left(f(p), \varepsilon_{0}\right)$, but this is obviously impossible. Hence $g(V) \not \subset$ $K\left(f(p), \varepsilon_{0}\right)$. We have thus shown $p \notin Q_{g}$.

It is easily seen that

$$
Q_{g} \subset F \text { and } Q_{g} \subset Q_{f} \cup\{p\} \cup(\mathbb{R} \backslash F) .
$$

Since $p \notin Q_{g}$, by (11), $Q_{g} \subset Q_{f}$. Since $Q_{f} \subset Q_{g}, Q_{f}=Q_{g}$. 
Now, we shall show that $C_{g}=C_{f}$. Obviously, $C_{f} \subset C_{g}$. Since $C_{g} \subset Q_{g}$, we conclude from (11) that $C_{g} \subset F$. The obvious inclusion $C_{g} \subset C_{f} \cup(\mathbb{R} \backslash F)$ gives $C_{g} \subset C_{f}$, which proves $C_{g}=C_{F}$. Since $Q_{g}=Q_{f}$ and since $C_{g}=C_{f}$, by (6), and (7)we can infer, in this case, that

$g$ is a Darboux function that is an $\varepsilon$-extension of $f$ such that

$$
Q_{g}=Q_{f} \text { and } C_{g}=C_{f} \text {. }
$$

$2^{0} p \in Q_{f}$.

Let $p_{n}=p+\frac{1}{n}$ for $n \geq 1$. Let us also put $P_{1}=\left[p_{1},+\infty\right)$ and $P_{n}=\left[p_{n}, p_{n-1}\right)$ for $n \geq 2$. In each $P_{n}$ we define the equivalence the relation $\star$ by

$$
x \star y \Longleftrightarrow x-y \in \mathbb{Q} .
$$

Let $\mathcal{P}_{n}$ denote the set of abstract classes of relation $\star$ for $n \geq 1$. Then, for $n \geq 1$, there exists a bijection $g_{n}: \mathcal{P}_{n} \stackrel{\text { onto }}{\longrightarrow} K\left(f(p), \frac{\varepsilon}{n}\right) \cap \Phi$ such that $g_{n}\left(\left[p_{n}\right]_{\star}\right) \in K\left(f(p), \frac{\varepsilon}{n+1}\right) \cap \Phi$. Then we define $g: \mathbb{R} \rightarrow \mathbb{R}^{2}$ by

$$
g(x)= \begin{cases}g_{n}\left([x]_{\star}\right) & x \text { if } \in P_{n}, n=1,2, \ldots, \\ f(x) & \text { if } x \in F\end{cases}
$$

Obviously,

$$
g \text { is an } \varepsilon \text {-extension of } f \text {. }
$$

We shall show that $g$ is a Darboux function. Let $C \subset \mathbb{R}^{2}$ be a connected set. Consider the following cases.

1) $C \subset F$; then, clearly, $g(C)=f(C)$ is connected.

2) $C \subset \mathbb{R} \backslash F$.

2a) $C$ is a point, so is $g(C)$.

2b) $C$ is a non-degenerate segment or a half-line.

Let $n_{0}=\min \left\{n \geq 1: C \cap P_{n} \neq \emptyset\right\}$.

$\left.2 b_{1}\right)$ If $C \cap P_{n_{0}} \neq\left\{p_{n_{0}}\right\}$, then $g\left(C \cap P_{n_{0}}\right)=g_{n_{0}}\left(\mathcal{P}_{n_{0}}\right)=K\left(f(p), \frac{\varepsilon}{n_{0}}\right) \cap \Phi$. Hence $g(C)=\left(K\left(f(p), \frac{\varepsilon}{n_{0}}\right) \cap \Phi\right) \cup \bigcup_{n=n_{0}+1}^{+\infty} g\left(P_{n} \cap C\right)=K\left(f(p), \frac{\varepsilon}{n_{0}}\right) \cap \Phi$.

$\left.2 b_{2}\right)$ If $C \cap P_{n_{0}}=\left\{p_{n_{0}}\right\}$, then $g\left(P_{n_{0}+1} \cap C\right)=g_{n_{0}+1}\left(\mathcal{P}_{n_{0}+1}\right)=K\left(f(p), \frac{\varepsilon}{n_{0}+1}\right) \cap$ $\Phi$. Hence $g(C)=\left(K\left(f(p), \frac{\varepsilon}{n_{0}+1}\right) \cap \Phi\right) \cup g_{n_{0}}\left(\left[p_{n_{0}}\right]_{\star}\right)=K\left(f(p), \frac{\varepsilon}{n_{0}+1}\right) \cap \Phi$.

Thus, in the latter two cases, $\mathrm{g}(\mathrm{C})$ is a connected set. 
3) $C \cap F \neq \emptyset$ and $C \backslash F \neq \emptyset$.

Notice that

$g(C \cap F)$ and $g(C \backslash F)$ are non-empty connected and non-separated sets.

Since $g(C)=g(C \cap F) \cup g(C \backslash F)$, it follows from (14) that $g(C)$ is connected. This completes the proof that $g$ is Darboux.

Now, we shall show that $Q_{g}=Q_{f}$. Obviously, $Q_{f} \subset Q_{g}$. To prove the inverse inclusion, let us note that $Q_{g} \subset F$ and $Q_{g} \subset Q_{f} \cup\{p\} \cup(\mathbb{R} \backslash F)$. Since $p \in Q_{f}, Q_{g} \subset Q_{f}$. This proves $Q_{g}=Q_{f}$.

We shall now show that $C_{g}=C_{f}$. For this purpose, it suffices to prove that $p \in C_{f} \Longleftrightarrow p \in C_{g}$. It is obvious that if $p \notin C_{f}$, then $p \notin C_{g}$. So, let us suppose that $p \in C_{f}$. Fix $\varepsilon^{\star}>0$. Choose $n_{0} \geq 1$ such that $\frac{\varepsilon}{n_{0}}<\varepsilon^{\star}$ and $\delta_{1}>0$ for which $f\left(\left(p-\delta_{1}, p\right]\right) \subset K\left(f(p), \varepsilon^{\star}\right)$. Set $\delta_{2}=p_{n_{0}}-p$ and $\delta=\min \left(\delta_{1}, \delta_{2}\right)$. Then

$$
g((p-\delta, p+\delta)) \subset K\left(f(p), \varepsilon^{\star}\right) .
$$

Therefore $p \in C_{g}$ and $C_{g}=C_{f}$ is proved.From The preceding we can infer, in this case, that

$$
\begin{aligned}
& g \text { is a Darboux function that is an } \varepsilon \text {-extension of } f \text { such that } \\
& Q_{g}=Q_{f} \text { and } C_{g}=C_{f} .
\end{aligned}
$$

By (12) and (15) to complete the proof, it suffices to show that, in cases $1^{0}$ and $2^{0}, g$ has a finite variation. To this end, note that

$$
N_{g}(y)= \begin{cases}N_{f}(y) & y \text { if } \notin K(f(p), \varepsilon) \cap \Phi, \\ +\infty & y \text { if } \in K(f(p), \varepsilon) \cap \Phi .\end{cases}
$$

Hence $N_{g}(y)=N_{f}(y)$ almost everywhere in $\mathbb{R}^{2}$. Therefore the measurability of $N_{f}$ implies that of $N_{g}$, and

$$
\begin{aligned}
\bigvee_{\mathbb{R}}(g) & =\int_{\mathbb{R}^{2}} N_{g}(y) d y=\int_{K(f(p), \varepsilon) \cap \Phi} N_{g}(y) d y+\int_{\mathbb{R}^{2} \backslash(K(f(p), \varepsilon) \cap \Phi)} N_{g}(y) d y \\
& =\int_{\mathbb{R}^{2} \backslash(K(f(p), \varepsilon) \cap \Phi)} N_{g}(y) d y=\int_{\mathbb{R}^{2} \backslash(K(f(p), \varepsilon) \cap \Phi)} N_{f}(y) d y \\
& \leq \int_{\mathbb{R}^{2}} N_{f}(y) d y=\bigvee_{\mathbb{R}}(f)<+\infty
\end{aligned}
$$


In a similar way one can show that there exists a function $g$ satisfying the conditions of the assertion in the case when $F$ is a non-degenerate segment. It is easy to consider the case when $F$ is a point.

Now we shall prove the main theorem.

Theorem 2. Let $F$ be a closed convex and boundary subset of $\mathbb{R}^{2}$. Let $f$ : $F \rightarrow \mathbb{R}^{2}$ be a Darboux function with finite variation. Then, for any $\varepsilon>0$, there exists a Darboux function $f^{\star}: \mathbb{R}^{2} \rightarrow \mathbb{R}^{2}$ with finite variation which is an $\varepsilon$-extension of $f$ such that $Q_{f^{\star}}=Q_{f}$ and $C_{f^{\star}}=C_{f}$.

Proof.By Lemma 2, we can consider only the case when $\mathrm{F}$ is a line. Let $H_{1}^{(1)}$ denote a closed half-line which is perpendicular to $F$, with endpoint $k_{1}^{(1)} \in F$. Let $L_{1}^{(1)}$ be a line containing $H_{1}^{(1)}$. Next, let $\Pi^{+}$stand for a closed half-plane with edge $F$, which contains $H_{1}^{(1)}$. For each $\alpha \in(0,+\infty)$, denote by $A_{\alpha} \subset \Pi^{+}$ the line which is parallel to $F$ and distant from $F$ by $\alpha$. Then let us denote $\left\{m_{1}^{1(1)}\right\}=A_{1} \cap H_{1}^{(1)}$. Let $H_{1}^{\prime},(1), H^{\prime \prime}{ }_{1}^{(1)}$ stand for two open half- lines lying on the opposite sides of the line $L_{1}^{(1)}$, with endpoint $m_{1}^{1(1)}$ included in $\bigcup_{\alpha>1} A_{\alpha}$, and let $P_{0}^{(1)}$ denote a closed convex set which is determined by ${H^{\prime}}_{1}^{(1)}$, a segment $\left[m_{1}^{1(1)}, k_{1}^{(1)}\right]$ and a half-line with endpoint $k_{1}^{(1)}$ included in $F$, which is on the same side of $L_{1}^{(1)}$ as $H_{1}^{,(1)}$.

Let $P_{1}^{(1)}$ be a closed convex set determined by $H_{1}^{,,(1)}$, a segment $\left[m_{1}^{1(1)}, k_{1}^{(1)}\right]$ and a half-line with endpoint $k_{1}^{(1)}$ included in $F$, which is on the same side of $L_{1}^{(1)}$ as $H_{1}^{\prime \prime}{ }_{1}^{(1)}$. Moreover, let $P_{1}^{\prime(1)}$ denote an open convex angle determined by $H_{1}^{\prime(1)}$ and $H^{\prime \prime}{ }_{1}^{(1)}$. Put $F_{0}^{(1)}=P_{0}^{(1)} \cap F, \quad F_{1}^{(1)}=P_{1}^{(1)} \cap F, \quad F_{0}^{,(1)}=$ $\bigcup_{y \in f\left(F_{0}^{(1)}\right)} K(y, \varepsilon)$ and $F_{1}^{,(1)}=\bigcup_{y \in f\left(F_{1}^{(1)}\right)} K(y, \varepsilon)$. For each $w \in(0,+\infty)$, let $\left\{\hat{m}_{w}^{1(1)}\right\}=H_{1}^{(1)} \cap A_{1+w}$ and let $H_{1, w}^{,(1)}\left(H_{1, w}^{,,(1)}\right)$ denote a closed half-line with endpoint $\hat{m}_{w}^{1(1)}$ which is parallel to $H_{1}^{,(1)}\left(H_{1}^{,,(1)}\right)$, included in $\Pi^{+}$. Moreover, set $K_{w}^{1(1)}=H_{1, w}^{,{ }^{(1)}} \cup H_{1, w}^{,(1)}$ for $w \in(0,+\infty)$. Note that, for each $x \in P_{1}^{,(1)}$, there exists only one $w \in(0,+\infty)$ such that $x \in K_{w}^{1(1)}$. Put $\mathcal{K}_{1}^{(1)}=\left\{K_{w}^{1(1)}\right.$ : $w \in(0,+\infty)\}$. In this family we define the equivalence relation $\circ$ by

$$
K_{w}^{1(1)} \circ K_{w^{\prime}}^{1(1)} \Longleftrightarrow w-w^{\prime} \in \mathbb{Q} .
$$

Let $\hat{\mathcal{K}}_{1}^{(1)}$ stand for the set of abstract classes of the relation $\circ$. Then there exists a bijection

$$
\psi_{1}^{(1)}: \hat{\mathcal{K}}_{1}^{(1)} \stackrel{\text { onto }}{\longmapsto}\left(F_{0}^{,(1)} \cup F_{1}^{,(1)}\right) \cap \Phi .
$$

Put $\mathcal{A}_{t}^{(1)}=\left\{A_{\alpha} \cap P_{t}^{(1)}: \alpha>1\right\}$ for $t=0,1$. In each of these families we define 
the equivalence relation $\diamond$ by

$$
\left(A_{\alpha^{\prime}} \cap P_{t}^{(1)}\right) \diamond\left(A_{\alpha^{\prime \prime}} \cap P_{t}^{(1)}\right) \Longleftrightarrow \alpha^{\prime}-\alpha^{\prime \prime} \in \mathbb{Q} .
$$

For $t=0,1$, let $\hat{\mathcal{A}}_{t}^{(1)}$ denote the set of abstract classes of the relation $\diamond$. Then there exist bijections

$$
\varphi_{0}^{(1)}: \hat{\mathcal{A}}_{0}^{(1)} \stackrel{\text { onto }}{\longrightarrow} F_{0}^{,(1)} \cap \Phi
$$

and

$$
\varphi_{1}^{(1)}: \hat{\mathcal{A}}_{1}^{(1)} \stackrel{\text { onto }}{\longrightarrow} F_{1}^{(1)} \cap \Phi .
$$

We define $g_{1}: \bigcup_{\alpha>1} A_{\alpha} \rightarrow \mathbb{R}^{2}$ by

$$
g_{1}(x)= \begin{cases}\psi_{1}^{(1)}\left(\left[K_{1}(x)\right]_{\diamond}\right) & \text { if } x \in P_{1}^{(1)} \\ \varphi_{0}^{(1)}\left(\left[A_{0}(x)\right]_{\diamond}\right) & \text { if } x \in P_{0}^{(1)} \cap \bigcup_{\alpha>1} A_{\alpha} \\ \varphi_{1}^{(1)}\left(\left[A_{1}(x)\right]_{\diamond}\right) & \text { if } x \in P_{1}^{(1)} \cap \bigcup_{\alpha>1} A_{\alpha}\end{cases}
$$

where $K_{1}(x)\left(A_{t}(x), t=0,1\right)$ denotes a set from the family $\mathcal{K}_{1}^{(1)}\left(\mathcal{A}_{t}^{(1)}, t=\right.$ $0,1)$, containing $x$. Now let $k_{1}^{(2)}, k_{3}^{(2)} \in F$ denote distinct points having distance 1 from $k_{1}^{(1)}$ and set $k_{2}^{(2)}=k_{1}^{(1)}$. Let $H_{1}^{(2)}, H_{2}^{(2)}, H_{3}^{(2)} \subset \Pi^{+}$be closed half-lines which are perpendicular to $F$, with endpoints $k_{1}^{(2)}, k_{2}^{(2)}, k_{3}^{(2)}$, respectively. Note that $H_{2}^{(2)}=H_{1}^{(1)}$. Set $\left\{m_{t}^{n(2)}\right\}=A_{\frac{1}{n}} \cap H_{t}^{(2)}, t=1,2,3$, $n=1,2$. Note that $m_{2}^{1(2)}=m_{1}^{1(1)}$. Let $L_{t}^{(2)}$ denote a line which contains $H_{t}^{(2)}$, $t=1,2,3$ and let $H_{t}^{,(2)}, H_{t}^{,{ }^{,(2)}} \subset \Pi^{+}(t=1,2,3)$ be closed half- lines lying on the opposite sides of the line $L_{t}^{(2)}$ with endpoint $m_{t}^{2(2)}$, such that the following conditions hold:

$$
\begin{gathered}
H_{1}^{,{ }^{,}(2)} \cap\left(m_{1}^{1(2)}, m_{2}^{1(2)}\right) \neq \emptyset, \quad H_{2}^{,(2)} \cap\left(m_{1}^{1(2)}, m_{2}^{1(2)}\right) \neq \emptyset, \\
H_{2}^{,{ }^{,(2)}} \cap\left(m_{2}^{1(2)}, m_{3}^{1(2)}\right) \neq \emptyset, \quad H_{3}^{,(2)} \cap\left(m_{2}^{1(2)}, m_{3}^{1(2)}\right) \neq \emptyset, \\
H_{1}^{,(2)}, H_{3}^{,,(2)} \text { are not parallel to } \mathrm{F}, \\
H_{t}^{,,(2)} \cap H_{t+1}^{,(2)} \subset \bigcup_{\alpha>1} A_{\alpha}(t=1,2) .
\end{gathered}
$$

Then let $P_{t}^{(2)}(t=1,2,3)$ denote an open convex angle between the half-lines $H_{t}^{,(2)}$ and $H_{t}^{,{ }^{,}(2)}$. Let $P_{t}^{(2)}(t=1,2)$ stand for a closed convex set determined by the half-line $H_{t}^{,{ }^{\prime}(2)}$, the segments $\left[m_{t}^{2(2)}, k_{t}^{(2)}\right],\left[k_{t}^{(2)}, k_{t+1}^{(2)}\right]$ and $\left[k_{t+1}^{(2)}, m_{t+1}^{(2)}\right]$ and the half-line $H_{t+1}^{(2)}$. Let $P_{0}^{(2)}$ denote a closed convex set determined by the halfline $H_{1}^{(2)}$, the segment $\left[m_{1}^{2(2)}, k_{1}^{(2)}\right]$ and a half-line with endpoint $k_{1}^{(2)}$, included in $F$, that does not contain $k_{2}^{(2)}$. Let $P_{3}^{(2)}$ be a closed convex set determined 
by the half-line $H_{3}^{,{ }^{,}(2)}$, the segment $\left[m_{3}^{2(2)}, k_{3}^{(2)}\right]$ and a half-line with endpoint $k_{3}^{(2)}$, included in $F$, that does not contain $k_{2}^{(2)}$. Put $F_{t}^{(2)}=P_{t}^{(2)} \cap F$ and $F_{t}^{,(2)}=\bigcup_{y \in f\left(F_{t}^{(2)}\right)} K\left(y, \frac{\varepsilon}{2}\right)$ for $t=0,1,2,3$.

For $w \in\left[0, \frac{1}{4}\right)$ and $t=1,2,3$, let $\left\{\hat{m}_{w}^{t(2)}\right\}=H_{t}^{(2)} \cap A_{\frac{3}{4}-w}$ and let $H_{t, w}^{(2)}\left(H_{t, w}^{,{ }^{\prime}(2)}\right)$ denote a closed half-line which is parallel to $H_{t}^{(2)}\left(H_{t}^{,{ }^{,}(2)}\right)$, with endpoint $\hat{m}_{w}^{t(2)}$, included in $\Pi^{+}$. Set $\left\{q_{t, w}^{,(2)}\right\}=H_{t, w}^{,(2)} \cap A_{\frac{3}{4}+w},\left\{q_{t, w}^{,(2)}\right\}=H_{t, w}^{,,(2)} \cap A_{\frac{3}{4}+w}$ for $w \in\left[0, \frac{1}{4}\right)$ and $t=1,2,3$. For $t=1,2,3$ and $w \in\left(0, \frac{1}{4}\right)$, let

$$
K_{w}^{t(2)}=\left[\hat{m}_{w}^{t(2)}, q_{t, w}^{(2)}\right] \cup\left[q_{t, w}^{(2)}, q_{t, w}^{,,(2)}\right] \cup\left[q_{t, w}^{,,(2)}, \hat{m}_{w}^{t(2)}\right]
$$

and $K_{0}^{t(2)}=\left\{\hat{m}_{0}^{t(2)}\right\}$. Observe that, for $t=1,2,3$ we have for each $x \in$ $P_{t}^{,(2)} \cap \bigcup_{\frac{1}{2}<\alpha<1} A_{\alpha}$, there exists only one $w \in\left[0, \frac{1}{4}\right)$ such that $x \in K_{w}^{t(2)}$. Put $\mathcal{K}_{t}^{2}=\left\{K_{w}^{t(2)}: w \in\left[0, \frac{1}{4}\right)\right\}$ for $t=1,2,3$. In each of these families we define the equivalence relation $\circ$ by

$$
K_{w}^{t(2)} \circ K_{w^{\prime}}^{t(2)} \Longleftrightarrow w-w^{\prime} \in \mathbb{Q} .
$$

For $t=1,2,3$, let $\hat{\mathcal{K}}_{t}^{(2)}$ denote the set of abstract classes of the relation $\circ$. Then there exist bijections

$$
\psi_{t}^{(2)}: \hat{\mathcal{K}}_{t}^{(2)} \stackrel{\text { onto }}{\longrightarrow}\left(F_{t-1}^{,(2)} \cup F_{t}^{,(2)}\right) \cap \Phi
$$

for $t=1,2,3$. Put $\mathcal{A}_{\sqcup}^{(\in)}=\left\{\mathcal{A}_{\alpha} \cap \mathcal{P}_{\sqcup}^{(\in)}: \frac{\infty}{\epsilon}<\alpha<\infty\right\}$ for $t=0,1,2,3$. In each of these families we define the equivalence relation $\diamond$ by

$$
\left(A_{\alpha^{\prime}} \cap P_{t}^{(2)}\right) \diamond\left(A_{\alpha "} \cap P_{t}^{(2)}\right) \Longleftrightarrow \alpha^{\prime}-\alpha " \in \mathbb{Q} .
$$

For $t=0,1,2,3$, let $\hat{\mathcal{A}}_{t}^{(2)}$ denote the set of abstract classes of the relation $\diamond$. Then there exist bijections

$$
\varphi_{t}^{(2)}: \hat{\mathcal{A}}_{t}^{(2)} \stackrel{\text { onto }}{\longrightarrow} F_{t}^{,(2)} \cap \Phi
$$

for $t=0,1,2,3$. Let $\hat{P}_{t}^{,(2)}\left(\tilde{P}_{t}^{,(2)}\right)$ denote an open convex angle included in $P_{t}^{,(2)}$, determined by the half-lines $H_{t}^{,(2)}$ and $H_{t}^{(2)}\left(H_{t}^{,{ }^{,(2)}}\right.$ and $\left.H_{t}^{(2)}\right)$ for $t=1,2,3$. Let us set

$$
\begin{array}{lcl}
A_{1}^{,(2)}= & A_{1} \cap\left(P_{0}^{(2)} \cup \hat{P}_{1}^{,(2)}\right), & A_{1}^{,(2)}=A_{1} \cap\left(\tilde{P}_{1}^{,(2)} \cup P_{1}^{(2)}\right), \\
A_{2}^{,(2)}= & A_{1} \cap \hat{P}_{2}^{,(2)}, & A_{2}^{,(2)}=A_{1} \cap\left(\tilde{P}_{2}^{,(2)} \cup P_{2}^{(2)}\right), \\
A_{3}^{,(2)}= & A_{1} \cap \hat{P}_{3}^{,(2)}, & A_{1}^{,(2)}=A_{1} \cap\left(\tilde{P}_{3}^{,(2)} \cup P_{3}^{(2)}\right) .
\end{array}
$$


In each of the sets $A_{t}^{(2)}$ and $A_{t}^{,(2)}(t=1,2,3)$ we define the equivalence relation $\star$ by

$$
x \star y \Longleftrightarrow \rho(x, y) \in \mathbb{Q}
$$

where $\rho$ denotes the metric in $\mathbb{R}^{2}$. For $t=1,2,3$, let $\hat{\mathcal{A}}_{t}^{,(2)}$ and $\hat{\mathcal{A}}_{t}^{,(2)}$ denote abstract classes of the relation $\star$. Then, for $t=1,2,3$, there exist bijections

$$
\xi_{t}^{,(2)}: \hat{\mathcal{A}}_{t}^{,(2)} \stackrel{\text { onto }}{\longrightarrow} F_{t-1}^{,(2)} \cap \Phi \quad \text { and } \quad \xi_{t}^{,(2)}: \hat{\mathcal{A}}_{t}^{,(2)} \stackrel{\text { onto }}{\longrightarrow} F_{t}^{,(2)} \cap \Phi .
$$

We define $g_{2}: \bigcup_{\frac{1}{2}<\alpha \leq 1} A_{\alpha} \rightarrow \mathbb{R}^{2}$ by

$$
g_{2}(x)= \begin{cases}\varphi_{t}^{(2)}\left(\left[A_{t}(x)\right]_{\diamond}\right) & \text { if } x \in P_{t}^{(2)} \cap \bigcup_{\frac{1}{2}<\alpha<1} A_{\alpha}, t=0,1,2,3, \\ \psi_{t}^{(2)}\left(\left[K_{t}(x)\right]_{\diamond}\right) & \text { if } x \in P_{t}^{,(2)} \cap \bigcup_{\frac{1}{2}<\alpha<1} A_{\alpha}, t=1,2,3 \\ \xi_{t}^{,(2)}\left([x]_{\star}\right) & \text { if } x \in A_{t}^{,(2)}, t=1,2,3 \\ \xi_{t}^{,,(2)}\left([x]_{\star}\right) & \text { if } x \in A_{t}^{,,(2)}, t=1,2,3 \\ f\left(k_{t}^{(2)}\right) & \text { if } x=m_{t}^{1(2)}, t=1,2,3\end{cases}
$$

where $A_{t}(x)\left(K_{t}(x)\right)$ denotes a set from the family $\mathcal{A}_{t}^{(2)}\left(\mathcal{K}_{t}^{(2)}\right)$, containing $x$. To define a function $g_{3}: \bigcup_{\frac{1}{3}<\alpha \leq \frac{1}{2}} A_{\alpha} \rightarrow \mathbb{R}^{2}$, let us draw four more half-lines contained in $\Pi^{+}$and perpendicular to $F$. The endpoints of these half-lines are points lying on $F$ which are the midpoints of the segments $\left[k_{1}^{(2)}, k_{2}^{(2)}\right]$ and $\left[k_{2}^{(2)}, k_{3}^{(2)}\right]$ or having distance 1 from the ends of the segment $\left[k_{1}^{(2)}, k_{3}^{(2)}\right]$ and do not belong to this segment. Thus we shall consider the points $k_{1}^{(3)}, \ldots, k_{7}^{(3)}$ (where $k_{2}^{(3)}=k_{1}^{(2)}, k_{4}^{(3)}=k_{2}^{(2)}=k_{1}^{(1)}, k_{6}^{(3)}=k_{3}^{(2)}$ ) and the half-lines $H_{1}^{(3)}, \ldots, H_{7}^{(3)}$, corresponding to them, and analogously for the function $g_{2}$. Following the above construction, we define an infinite family of functions

$g_{n}: \bigcup_{\frac{1}{n}<\alpha \leq \frac{1}{n-1}} A_{\alpha} \rightarrow \mathbb{R}^{2}(n=2,3, \ldots)$.

Then we define $g: \Pi^{+} \rightarrow \mathbb{R}^{2}$ by

$$
g(x)= \begin{cases}f(x) & \text { if } x \in F \\ g_{n}(x), & \text { if } x \in \bigcup_{\frac{1}{n}<\alpha \leq \frac{1}{n-1}} A_{\alpha}, n=2,3, \ldots, \\ g_{1}(x) & \text { if } x \in \bigcup_{\alpha>1} A_{\alpha} .\end{cases}
$$

We shall now prove some facts which will be used in the latter part of the 
proof. We shall first show that

if $C \subset \mathbb{R}^{2}$ is a connected set such that $C \cap P_{t_{0}}^{\left(n_{0}\right)} \cap \bigcup_{\frac{1}{n_{0}}<\alpha<\frac{1}{n_{0}-1}} A_{\alpha} \neq \emptyset$

for some $n_{0}>1$ and $t_{0} \in \mathbb{N}$ (for which $P_{t}^{,\left(n_{0}\right)}$ is defined) and

$C \not \subset K_{w}^{t_{0}\left(n_{0}\right)}$ for any $w \in\left[0, \frac{1}{2 n_{0}\left(n_{0}-1\right)}\right)$,

then $\left(F_{t_{0}-1}^{,\left(n_{0}\right)} \cup F_{t_{0}}^{,\left(n_{0}\right)}\right) \cap \Phi \subset g(C)$.

In order to simplify the notation, let $n_{0}=2, t_{0}=1$ and $P=P_{1}^{(2)} \cap$ $\bigcup_{\frac{1}{2}<\alpha<1} A_{\alpha}$. Then there exists $a_{0} \in C \cap P$. It follows that $a_{0} \in K_{w_{0}}^{1(2)}$ for some $w_{0} \in\left[0, \frac{1}{4}\right)$. Note that

$$
(\star) \quad \exists_{w_{1} \in\left[0, \frac{1}{4}\right)}\left(w_{1} \neq w_{0} \quad \wedge \quad C \cap K_{w_{1}}^{1(2)} \neq \emptyset\right) .
$$

Indeed, if, for any $w \neq w_{0}$ and $w \in\left[0, \frac{1}{4}\right), C \cap K_{w}^{1(2)}=\emptyset$, then there exists $b \in C \backslash P$. Let $w^{\prime} \in\left(w_{0}, \frac{1}{4}\right)$. Then $K_{w^{\prime}}^{1(2)} \cap C=\emptyset$ and $K_{w^{\prime}}^{1(2)}$ cuts the plane between $a_{0} \in C$ and $b \in C$, which contradicts the connectedness of C. We have thus proved $(\star)$.

So, there exists $a_{1} \in C \cap K_{w_{1}}^{1(2)}$. Assume that $w_{0}<w_{1}$. Notice that, for $w \in\left(w_{0}, w_{1}\right), K_{w}^{1(2)}$ cuts the plane between $a_{0}$ and $a_{1}$, so $C \cap K_{w}^{1(2)} \neq \emptyset$ for any $w \in\left(w_{0}, w_{1}\right)$. Therefore, in each abstract class of $\hat{\mathcal{K}}_{1}^{(2)}$, there exists a set $\mathrm{K}$ such that $C \cap K \neq \emptyset$. Consequently, by the definition of $g$, we obtain

$$
g(C \cap P)=g_{2}\left(\hat{\mathcal{K}}_{1}^{(2)}\right)=\left(F_{0}^{,(2)} \cup F_{1}^{,(2)}\right) \cap \Phi .
$$

Hence $\left(F_{0}^{,(2)} \cup F_{1}^{,(2)}\right) \cap \Phi \subset g(C)$, which proves (16). Similarly one can prove that

if $C \subset \mathbb{R}^{2}$ is a connected set suchthat $C \cap P_{1}^{,(1)} \neq \emptyset$

and $C \not \subset K_{w}^{1(1)}$ for any $w \in(0,+\infty)$, then $\left(F_{0}^{,(1)} \cup F_{1}^{,(1)}\right) \cap \Phi \subset g(C)$.

Now, we shall show that

if $C \subset \mathbb{R}^{2}$ is a connected set such that $C \cap P_{t_{0}}^{\left(n_{0}\right)} \cap \bigcup_{\frac{1}{n_{0}}<\alpha<\frac{1}{n_{0}-1}} A_{\alpha} \neq \emptyset$

for some $n_{0}>1$ and $t_{0} \in \mathbb{N}$ (for which $P_{t}^{\left(n_{0}\right)}$ is defined) and

$C \not \subset A_{\alpha} \cap P_{t_{0}}^{\left(n_{0}\right)}$ for any $\alpha \in\left(\frac{1}{n_{0}}, \frac{1}{n_{0}-1}\right)$, then $F_{t_{0}}^{,\left(n_{0}\right)} \cap \Phi \subset g(C)$. 
To simplify the notation, let $n_{0}=2, t_{0}=1$ and $R=P_{1}^{(2)} \cap \bigcup_{\frac{1}{2}<\alpha<1} A_{\alpha}$. There exists $a_{0} \in C \cap R$, and then $a_{0} \in A_{\alpha_{0}}$ for some $\alpha_{0} \in\left(\frac{1}{2}, 1\right)$. Note that

$$
(\star \star) \quad\left[C \backslash\left(A_{\alpha_{0}} \cap P_{1}^{(2)}\right)\right] \cap\left(P_{1}^{,(2)} \cup P_{1}^{(2)} \cup P_{2}^{,(2)}\right) \cap \bigcup_{\frac{1}{2}<\alpha<1} A_{\alpha} \neq \emptyset .
$$

If $C \cap\left(P_{1}^{,(2)} \cup P_{2}^{,(2)}\right) \cap \bigcup_{\frac{1}{2}<\alpha<1} A_{\alpha} \neq \emptyset$, then from (16) it follows that $F_{1}^{,(2)} \cap \Phi \subset$ $g(C)$, which gives (18). So now, let us assume that

$$
C \cap\left(P_{1}^{,(2)} \cup P_{2}^{,(2)}\right) \cap \bigcup_{\frac{1}{2}<\alpha<1} A_{\alpha}=\emptyset .
$$

By $(\star \star)$, we have $\left[C \backslash\left(A_{\alpha_{0}} \cap P_{1}^{(2)}\right)\right] \cap R \neq \emptyset$, hence $(C \cap R) \backslash A_{\alpha_{0}} \neq \emptyset$. So, there exists $a_{1} \in(C \cap R) \backslash A_{\alpha_{0}}$, thus $a_{1} \in A_{\alpha_{1}} \cap P_{1}^{(2)}$ for some $\alpha_{1} \in\left(\frac{1}{2}, 1\right)$ and $\alpha_{1} \neq \alpha_{0}$. We can assume that $\alpha_{0}<\alpha_{1}$. Then $g(C \cap R)=F_{1}^{,(2)} \cap \Phi$. From the above considerations we obtain the inclusion $F_{1}^{,(2)} \cap \Phi \subset g(C)$, which yields (18). Similarly one can prove that

if $C \subset \mathbb{R}^{2}$ is a connected set such that $C \cap P_{t_{0}}^{(1)} \cap \bigcup_{\alpha>1} A_{\alpha} \neq \emptyset$

for some $t_{0} \in\{0,1\}$ and $C \not \subset A_{\alpha} \cap P_{t_{0}}^{(1)}$ for any $\alpha>1$

then $F_{t_{0}}^{,(1)} \cap \Phi \subset g(C)$.

Now, we show that:

if $C \subset \mathbb{R}^{2}$ is a connected set which contains at least two

distinct points, such that $C \cap A_{t_{0}}^{,\left(n_{0}\right)} \neq \emptyset$ for some $n_{0} \geq 2$ and

$t_{0} \geq 1$ (for which $A_{t}^{,\left(n_{0}\right)}$ is defined), then $F_{t_{0}-1}^{,\left(n_{0}\right)} \cap \Phi \subset g(C)$.

For simplicity, let $n_{0}=2$. By our assumption, there exists $a_{0} \in C \cap A_{t_{0}}^{(2)}$. Consider the following cases.

$1^{0}: t_{0} \in\{2,3\}$.

We give the proof only for the case $t_{0}=2$ (the other casebeingsimilar). Notice that

$$
(\star \star \star) \quad C \cap\left[\left(A_{2}^{,(2)} \backslash\left\{a_{0}\right\}\right) \cup\left(P_{2}^{,(2)} \cap \bigcup_{\frac{1}{2}<\alpha<1} A_{\alpha}\right) \cup\left(P_{0}^{(1)} \cap \bigcup_{\alpha>1} A_{\alpha}\right)\right] \neq \emptyset .
$$

Then there are two possible cases.

1): $C \cap\left[\left(P_{2}^{,(2)} \cap \bigcup_{\frac{1}{2}<\alpha<1} A_{\alpha}\right) \cup\left(P_{0}^{(1)} \cap \bigcup_{\alpha>1} A_{\alpha}\right)\right] \neq \emptyset$. 
Then $C \cap P_{2}^{(2)} \cap \bigcup_{\frac{1}{2}<\alpha<1} A_{\alpha} \neq \emptyset$ or $C \cap P_{0}^{(1)} \cap \bigcup_{\alpha>1} A_{\alpha} \neq \emptyset$. If $C \cap P_{2}^{,(2)} \cap$ $\bigcup_{\frac{1}{2}<\alpha<1} A_{\alpha} \neq \emptyset$, then the assumptions of condition (16) hold (for $t_{0}=2, n_{0}=$ 2), so $F_{1}^{,{ }^{(2)}} \cap \Phi \subset\left(F_{1}^{,{ }^{(2)}} \cup F_{2}^{,(2)}\right) \cap \Phi \subset g(C)$. If $C \cap P_{0}^{(1)} \cap \bigcup_{\alpha>1} A_{\alpha} \neq \emptyset$ then the assumptions of condition (19) hold (for $t_{0}=0$ ), so $F_{1}^{,{ }^{(2)}} \cap \Phi \subset F_{0}^{,(1)} \cap \Phi \subset g(C)$ (because $F_{1}^{(2)} \subset F_{0}^{(1)}$ ).

2): $C \cap\left[\left(P_{2}^{,(2)} \cap \bigcup_{\frac{1}{2}<\alpha<1} A_{\alpha}\right) \cup\left(P_{0}^{(1)} \cap \bigcup_{\alpha>1} A_{\alpha}\right)\right]=\emptyset$.

Then from $(\star \star \star)$ we have $C \cap\left(A_{2}^{,(2)} \backslash\left\{a_{0}\right\}\right) \neq \emptyset$. So, there exists $a_{1} \in$ $C \cap\left(A_{2}^{(2)} \backslash\left\{a_{0}\right\}\right)$. Notice that $C \cap A_{2}^{,(2)}$ contains some non-degenerate segment. From the definitions of $g_{2}$ and $g$ we have

$$
F_{1}^{,(2)} \cap \Phi \subset g_{2}\left(\hat{\mathcal{A}}_{2}^{,(2)}\right)=g\left(C \cap A_{2}^{,(2)}\right) \subset g(C) .
$$

Hence, in both cases, $F_{1}^{,(2)} \cap \Phi \subset g(C)$, which proves (20).

$2^{0} t_{0}=1$.

A similar analysis to that in case $1^{0}$ shows that

$$
\begin{aligned}
& C \cap\left[\left(A_{1}^{,(2)} \backslash\left\{a_{0}\right\}\right) \cup\left(P_{1}^{,(2)} \cap \bigcup_{\frac{1}{2}<\alpha<1} A_{\alpha}\right) \cup\left(P_{0}^{(1)} \cap \bigcup_{\alpha>1} A_{\alpha}\right)\right] \\
& \cup\left(P_{0}^{(2)} \cap \bigcup_{\frac{1}{2}<\alpha<1} A_{\alpha}\right) \neq \emptyset
\end{aligned}
$$

and, the remainder of the proof is analogously to that of case $1^{0}$. Condition (20) has thus been proved.

Now, we shall show that

if $C \subset \mathbb{R}^{2}$; is a connected set which contains at least two distinct points, such that $C \cap A_{t_{0}}^{,\left(n_{0}\right)} \neq \emptyset$ for some $n_{0} \geq 2$ and $t_{0} \geq 1$ (for which $A_{t}^{,\left(n_{0}\right)}$ is defined), then $F_{t_{0}}^{,\left(n_{0}\right)} \cap \Phi \subset g(C)$.

For simplicity, let $n_{0}=2$. By our assumption, there exists $a_{0} \in C \cap A_{t_{0}}^{,(2)}$. Let us consider the following cases.

$1^{0}: t_{0} \in\{1,2\}$.

We shall demonstrate the proof only for the case $t_{0}=1$ (the other case being 
similar). Let us notice that

$$
\begin{gathered}
(\star \star \star \star) C \cap\left[\left(A_{1}^{,(2)} \backslash\left\{a_{0}\right\}\right) \cup\left(P_{1}^{,(2)} \cap \bigcup_{\frac{1}{2}<\alpha<1} A_{\alpha}\right) \cup\left(P_{1}^{(2)} \cap \bigcup_{\frac{1}{2}<\alpha<1} A_{\alpha}\right)\right. \\
\left.\cup\left(P_{2}^{,(2)} \cap \bigcup_{\frac{1}{2}<\alpha<1} A_{\alpha}\right) \cup\left(P_{0}^{(1)} \cap \bigcup_{\alpha>1} A_{\alpha}\right) \cup A_{2}^{,(2)}\right] \neq \emptyset .
\end{gathered}
$$

Then two cases are possible.

$$
\begin{aligned}
1): C & \left.\cap\left(P_{1}^{,(2)} \cap \bigcup_{\frac{1}{2}<\alpha<1} A_{\alpha}\right) \cup\left(P_{1}^{(2)} \cap \bigcup_{\frac{1}{2}<\alpha<1} A_{\alpha}\right)\right] \\
& \cup\left(P_{2}^{,(2)} \cap \bigcup_{\frac{1}{2}<\alpha<1} A_{\alpha}\right) \cup\left(P_{0}^{(1)} \cap \bigcup_{\alpha>1} A_{\alpha}\right) \cup A_{2}^{,(2)} \neq \emptyset .
\end{aligned}
$$

Then

$$
\begin{aligned}
& C \cap P_{1}^{,(2)} \cap \bigcup_{\frac{1}{2}<\alpha<1} A_{\alpha} \neq \emptyset \text { or } C \cap P_{1}^{(2)} \cap \bigcup_{\frac{1}{2}<\alpha<1} A_{\alpha} \neq \emptyset \text { or } \\
& C \cap P_{2}^{,(2)} \cap \bigcup_{\frac{1}{2}<\alpha<1} A_{\alpha} \neq \emptyset \text { or } C \cap P_{0}^{(1)} \cap \bigcup_{\alpha>1} A_{\alpha} \neq \emptyset \text { or } \\
& C \cap A_{2}^{,(2)} \neq \emptyset
\end{aligned}
$$

If $C \cap P_{1}^{,(2)} \cap \bigcup_{\frac{1}{2}<\alpha<1} A_{\alpha} \neq \emptyset$, then the assumptions of condition (16) hold (for $t_{0}=1, n_{0}=2$ ), so $F_{1}^{,(2)} \cap \Phi \subset\left(F_{0}^{,(2)} \cup F_{1}^{,(2)}\right) \cap \Phi \subset g(C)$.

If $C \cap P_{1}^{(2)} \cap \bigcup_{\frac{1}{2}<\alpha<1} A_{\alpha} \neq \emptyset$, then the assumptions of condition (18) hold (for $\left.t_{0}=1, n_{0}=2\right)$, so $F_{1}^{,(2)} \cap \Phi \subset g(C)$.

If $C \cap P_{2}^{,(2)} \cap \bigcup_{\frac{1}{2}<\alpha<1} A_{\alpha} \neq \emptyset$, then from condition (16) we have $F_{1}^{,(2)} \cap \Phi \subset$ $\left(F_{1}^{,(2)} \cup F_{2}^{,(2)}\right) \cap \Phi \subset g(C)$.

If $C \cap P_{0}^{(1)} \cap \bigcup_{\alpha>1} A_{\alpha} \neq \emptyset$, then from condition (19) we get $F_{1}^{,(2)} \cap \Phi \subset$ $F_{0}^{,(1)} \cap \Phi \subset g(C)$.

If $C \cap A_{2}^{,(2)} \neq \emptyset$, then condition (20) gives $F_{1}^{,(2)} \cap \Phi \subset g(C)$.

2): $C \cap\left[\left(P_{1}^{,(2)} \cap \bigcup_{\frac{1}{2}<\alpha<1} A_{\alpha}\right) \cup\left(P_{1}^{(2)} \cap \bigcup_{\frac{1}{2}<\alpha<1} A_{\alpha}\right) \cup\left(P_{2}^{,(2)} \cap \bigcup_{\frac{1}{2}<\alpha<1} A_{\alpha}\right) \cup\right.$ $\left.\left(P_{0}^{(1)} \cap \bigcup_{\alpha>1} A_{\alpha}\right) \cup A_{2}^{(2)}\right] \neq \emptyset$.

Then from $(\star \star \star \star \star)$ we have $C \cap\left(A_{1}^{,,(2)} \backslash\left\{a_{0}\right\}\right) \neq \emptyset$. Consequently, there exists

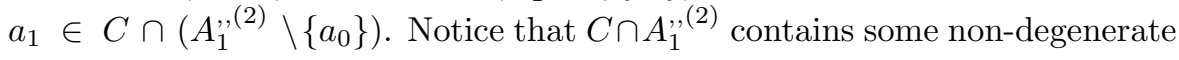


segment. By the definitions of $g_{2}$ and $g$,

$$
F_{1}^{,(2)} \cap \Phi \subset g_{2}\left(\hat{\mathcal{A}}_{1}^{,(2)}\right)=g\left(C \cap A_{1}^{,(2)}\right) \subset g(C) .
$$

Hence, in both cases, $F_{1}^{,(2)} \cap \Phi \subset g(C)$, which proves $(21)$.

$2^{0}: t_{0}=3$.

Similarly, we could first show that

$$
C \cap\left[\left(P_{3}^{,(2)} \cap \bigcup_{\frac{1}{2}<\alpha<1} A_{\alpha}\right) \cup\left(P_{3}^{(2)} \cap \bigcup_{\frac{1}{2}<\alpha<1} A_{\alpha}\right) \cup\left(P_{1}^{(1)} \cap \bigcup_{\alpha>1} A_{\alpha}\right)\right] \neq \emptyset
$$

and, the remainder of the proof is similar to that of case $1^{0}$ ). Condition (21) has thus been proved. Now, we shall show that

$$
g \text { is a Darboux function. }
$$

Let $C_{1}$ be a connected set. If $C_{1} \subset F$ or $C_{1}$ is a point, then, clearly, $g\left(C_{1}\right)$ is a connected set. The set $g\left(C_{1}\right)$ is a point (thus a connected set) in the following cases.

$$
\begin{array}{ll} 
& C_{1} \subset K_{w}^{t(n)} \text { for some } n, t \text { and } w \in\left[0, \frac{1}{2 n(n-1)}\right), \\
\text { or } \quad & C_{1} \subset K_{w}^{1(1)} \text { for some } w \in(0,+\infty), \\
\text { or } \quad & C_{1} \subset A_{\alpha} \cap P_{t}^{(n)} \text { for some } n, t \text { and } \alpha \in\left(\frac{1}{n}, \frac{1}{n-1}\right), \\
\text { or } \quad & C_{1} \subset A_{\alpha} \cap P_{t}^{(1)} \text { for some } t \text { and } \alpha>1 .
\end{array}
$$

Suppose that $g$ is not a Darboux function. Then there exists a connected set $\mathrm{C}$ such that $g(C)=W \cup T$ where $\mathrm{W}$ and $\mathrm{T}$ are non-empty and separated. Clearly, this does not occur for any cases which we considered for $C_{1}$. For each $n \in \mathbb{N}$, let $\backslash\{0\}, D_{0}^{(n)}\left(D_{2^{n}-1}^{(n)}\right)$ denote the intersection of the closed half-plane with edge $L_{1}^{(n)}\left(L_{2^{n}-1}^{(n)}\right)$, containing $H_{1}^{,(n)}\left(H_{2^{n}-1}^{,,(n)}\right)$, and the zone $\bigcup_{\frac{1}{n}<\alpha \leq \frac{1}{n-1}} A_{\alpha}$ (or, for $n=1: \bigcup_{\alpha>1} A_{\alpha}$ ). For any $n>1$ and $t \in\left\{1,2, \ldots, 2^{n}-2\right\}$, let $D_{t}^{(n)}$ denote the intersection of a closed zone between the lines $L_{t}^{(n)}$ and $L_{t+1}^{(n)}$ and of the zone $\bigcup_{\frac{1}{n}<\alpha \leq \frac{1}{n-1}} A_{\alpha}$. For $n \geq 2$, put $\hat{M}_{n}=\bigcup_{t=1}^{2^{n}-1}\left\{m_{t}^{n-1(n)}\right\}$. Let $M=\left\{n \geq 2: C \cap\left(\bigcup_{\frac{1}{n}<\alpha \leq \frac{1}{n-1}} A_{\alpha} \backslash \hat{M}_{n}\right) \neq \emptyset\right\} \quad$ if $C \cap \bigcup_{\alpha>1} A_{\alpha}=\emptyset \quad$ or $M=\left\{n \geq 2: C \cap\left(\bigcup_{\frac{1}{n}<\alpha \leq \frac{1}{n-1}} A_{\alpha} \backslash \hat{M}_{n}\right) \neq \emptyset\right\} \cup\{1\} \quad$ if $C \cap \bigcup_{\alpha>1} A_{\alpha} \neq \emptyset$. Observe that $M \neq \emptyset$. For each $n \in M$, let

$$
\mathcal{D}_{n}=\left\{D_{t_{i}}^{(n)}: i=1, \ldots, l_{n}\right\} \subset\left\{D_{t}^{(n)}: t \in\left\{0,1, \ldots, 2^{n}-1\right\}\right\},
$$


where $\left(t_{i}\right)_{i=1, \ldots, l_{n}}$ is a subsequence of the sequence $\left(0,1, \ldots, 2^{n}-1\right)$, be a family of sets such that $\left(C \cap D_{t_{i}}^{(n)}\right) \backslash\left\{m_{t_{i}}^{n-1(n)}, m_{t_{i}+1}^{n-1(n)}\right\} \neq \emptyset$ (if the points $m_{t_{i}}^{n-1(n)}, m_{t_{i}+1}^{n-1(n)}$ are defined). Note that, for each $n \in M, \mathcal{D}_{n} \neq \emptyset$. Let $n \in M$ and $i \in\left\{1, \ldots, l_{n}\right\}$. Let $n \neq 1$ (for $n=1$, the proof is similar). Moreover, let $i$ be a number such that $t_{i} \neq \emptyset$ and $t_{i} \neq 2^{n}-1$ (for $t_{i}=0$ or $t_{i}=2^{n}-1$, the proof is similar). Then, from the definition of $\mathcal{D}_{n}$ it follows that

$$
\begin{aligned}
& C \cap P_{t_{i}}^{,(n)} \cap \bigcup_{\frac{1}{n}<\alpha<\frac{1}{n-1}} A_{\alpha} \neq \emptyset \text { or } C \cap P_{t_{i}}^{(n)} \cap \bigcup_{\frac{1}{n}<\alpha<\frac{1}{n-1}} A_{\alpha} \neq \emptyset \\
& \text { or } C \cap P_{t_{i}+1}^{,(n)} \cap \bigcup_{\frac{1}{n}<\alpha<\frac{1}{n-1}} A_{\alpha} \neq \emptyset \text { or } C \cap A_{t_{i}}^{,(n)} \neq \emptyset \\
& \text { or } C \cap A_{t_{i}+1}^{,(n)} \neq \emptyset .
\end{aligned}
$$

If $C \cap P_{t_{i}}^{,(n)} \cap \bigcup_{\frac{1}{n}<\alpha<\frac{1}{n-1}} A_{\alpha} \neq \emptyset$, then, by the assumptions, from (16) we deduce that $F_{t_{i}}^{,(n)} \cap \Phi \subset\left(F_{t_{i}-1}^{,(n)} \cup F_{t_{i}}^{,(n)}\right) \cap \Phi \subset g(C)$.

If $C \cap P_{t_{i}}^{(n)} \cap \bigcup_{\frac{1}{n}<\alpha<\frac{1}{n-1}} A_{\alpha} \neq \emptyset$, then, by the assumptions, from (18) we conclude that $F_{t_{i}}^{,(n)} \cap \Phi \subset g(C)$.

If $C \cap P_{t_{i}+1}^{(n)} \cap \bigcup_{\frac{1}{n}<\alpha<\frac{1}{n-1}} A_{\alpha} \neq \emptyset$, then, by the assumptions, from (16) we infer that $F_{t_{i}}^{,(n)} \cap \Phi \subset\left(F_{t_{i}}^{(n)} \cup F_{t_{i}+1}^{,(n)}\right) \cap \Phi \subset g(C)$.

If $C \cap A_{t_{i}}^{,(n)} \neq \emptyset$, then, by the assumptions, from (21) we conclude that $F_{t_{i}}^{,(n)} \cap \Phi \subset g(C)$.

If $C \cap A_{t_{i}+1}^{(n)} \neq \emptyset$, then from (20) we deduce that $F_{t_{i}}^{,(n)} \cap \Phi \subset g(C)$.

Thus we have that the inclusion $F_{t_{i}}^{,(n)} \cap \Phi \subset g(C)$ holds for any $n \in M$ and $i \in\left\{1, \ldots, l_{n}\right\}$. This means that

$$
g(F \cap C) \cup \bigcup_{n \in M} \bigcup_{i=1}^{l_{n}}\left(F_{t_{i}}^{,(n)} \cap \Phi\right) \subset g(C) .
$$

Let $E=\bigcup_{n \in M} \bigcup_{i=1}^{l_{n}} F_{t_{i}}^{(n)}$. Note that $F_{t_{i}}^{(n)}=\operatorname{proj}_{F}\left(D_{t_{i}}^{(n)}\right)$ for any $n \in M$ and $i \in\left\{1, \ldots, l_{n}\right\}$. Moreover, observe that:

$$
(F \cap C) \cup E \text { is a connected set. }
$$

Since $f$ is a Darboux function and $(F \cap C) \cup E \subset F$, from (24) it follows that $f((F \cap C) \cup E)$ is a connected set. Then, also,

$$
Z=\bigcup_{n \in M} \bigcup_{i=1}^{l_{n}} F_{t_{i}}^{,(n)} \cup f(F \cap C) \text { is a connected set. }
$$


Indeed, note that

$$
Z=\bigcup_{n \in M} \bigcup_{i=1}^{l_{n}} F_{t_{i}}^{,(n)} \cup f((F \cap C) \cup E),
$$

because $f(E) \subset \bigcup_{n \in M} \bigcup_{i=1}^{l_{n}} F_{t_{i}}^{,(n)}$. Since, $f((F \cap C) \cup E), F_{t_{i}}^{,(n)}$ (for $n \in M$ and $\left.i \in\left\{1, \ldots, l_{n}\right\}\right)$ are connected, and $f((F \cap C) \cup E) \cap F_{t_{i}}^{,(n)} \supset f(E) \cap F_{t_{i}}^{,(n)} \supset$ $f\left(F_{t_{i}}^{(n)}\right) \cap F_{t_{i}}^{,(n)}=f\left(F_{t_{i}}^{(n)}\right) \neq \emptyset$. Therefore $Z$ is connected. From Lemma 1 and from (25) we deduce that

$$
\left(\bigcup_{n \in M} \bigcup_{i=1}^{l_{n}} F_{t_{i}}^{,(n)} \cap \Phi\right) \cup f(F \cap C) \text { is a connected set. }
$$

Since $g(C)=W \cup T, f(F \cap C)=g(F \cap C), W$ and $T$ are separated. Therefore from (23) and (26) we see that $g(F \cap C) \cup \bigcup_{n \in M} \bigcup_{i=1}^{l_{n}}\left(F_{t_{i}}^{,(n)} \cap \Phi\right) \subset W \quad$ or $g(F \cap C) \cup \bigcup_{n \in M} \bigcup_{i=1}^{l_{n}}\left(F_{t_{i}}^{,(n)} \cap \Phi\right) \subset T$. We may assume that

$$
g(F \cap C) \cup \bigcup_{n \in M} \bigcup_{i=1}^{l_{n}}\left(F_{t_{i}}^{,(n)} \cap \Phi\right) \subset W .
$$

(The other case is analogous.)

Now, we shall show that $g(C) \subset W$.Choose $x \in C$. If $x \in F$, then, by (27), $g(x) \in g(F \cap C) \subset W$. So, we assume that $x \notin F$. Then $x \in D_{t_{0}}^{\left(n_{0}\right)}$ for some $n_{0} \in M, t_{0} \in\left\{0,1, \ldots, 2^{n_{0}}-1\right\}$. Let $n_{0}>1$ (for $n_{0}=1$, the proof is similar) and let $t_{0} \neq 0, t_{0} \neq 2^{n_{0}}-1$, (for $t_{0}=0$ or $t_{0}=2^{n_{0}}-1$ the proof is similar). Consider the following three cases

$1^{0}: x \neq m_{t_{0}}^{n_{0}-1\left(n_{0}\right)}$ and $x \neq m_{t_{0}+1}^{n_{0}-1\left(n_{0}\right)}$.

Then $D_{t_{0}}^{\left(n_{0}\right)} \in \mathcal{D} \backslash$. From $(27)$ we have $F_{t_{0}}^{,\left(n_{0}\right)} \cap \Phi \subset W$.Consider the following subcases.

1a): $x \in P_{t_{0}}^{,\left(n_{0}\right)} \cap \bigcup_{\frac{1}{n_{0}}<\alpha<\frac{1}{n_{0}-1}} A_{\alpha}$.

Then $g(x) \in\left(F_{t_{0}-1}^{,\left(n_{0}\right)} \cup F_{t_{0}}^{,\left(n_{0}\right)}\right) \cap \Phi$. Since $x \in C$ and $x \in P_{t_{0}}^{,\left(n_{0}\right)} \cap \bigcup_{\frac{1}{n_{0}}<\alpha<\frac{1}{n_{0}-1}} A_{\alpha}$, we have $C \cap P_{t_{0}}^{,\left(n_{0}\right)} \cap \bigcup_{\frac{1}{n_{0}}<\alpha<\frac{1}{n_{0}-1}} A_{\alpha} \neq \emptyset$. Hence from (16) we infer that

$$
\left(F_{t_{0}-1}^{,\left(n_{0}\right)} \cup F_{t_{0}}^{,\left(n_{0}\right)}\right) \cap \Phi \subset g(C)=W \cup T
$$

From the connectedness of the set $\left(F_{t_{0}-1}^{,\left(n_{0}\right)} \cup F_{t_{0}}^{,\left(n_{0}\right)}\right) \cap \Phi$, the fact that the sets $W$ and $T$ are separated, the condition $\left(F_{t_{0}-1}^{,\left(n_{0}\right)} \cup F_{t_{0}}^{,\left(n_{0}\right)}\right) \cap \Phi \neq \emptyset$ and since 
$F_{t_{0}}^{,\left(n_{0}\right)} \cap \Phi \subset W$, it follows that $\left(F_{t_{0}-1}^{,\left(n_{0}\right)} \cup F_{t_{0}}^{,\left(n_{0}\right)}\right) \cap \Phi \subset W$. Hence $g(x) \in W$. 1b): $x \in P_{t_{0}+1}^{,\left(n_{0}\right)} \cap \bigcup_{\frac{1}{n_{0}}<\alpha \leq \frac{1}{n_{0}-1}} A_{\alpha}$.

This case is similar to 1a).

1c) $: x \in\left(P_{t_{0}}^{\left(n_{0}\right)} \cap \bigcup_{\frac{1}{n_{0}}<\alpha<\frac{1}{n_{0}-1}} A_{\alpha}\right) \cup A_{t_{0}}^{,\left(n_{0}\right)} \cup A_{t_{0}+1}^{,\left(n_{0}\right)}$.

Then from the definitions of the functions $g$ and $g_{n}$ and since $g(C) \subset W$, it follows that $g(x) \in F_{t_{0}}^{,\left(n_{0}\right)} \cap \Phi \subset W$.

$2^{0}: x=m_{t_{0}}^{n_{0}-1\left(n_{0}\right)}$.

Then :

- if $L_{t_{0}}^{\left(n_{0}\right)}=L_{\left.t_{1}-1\right)}^{\left(n_{0}-1\right)}$ for some $t_{1} \in\left\{0,1, \ldots, 2^{n_{0}-1}-1\right\}$, then at least one of the sets $D_{t_{0}-1}^{\left(n_{0}\right)}, D_{t_{0}}^{\left(n_{0}\right)}, D_{t_{1}-1}^{\left(n_{0}-1\right)}, D_{t_{1}}^{\left(n_{0}-1\right)}$ belongs to the family $\left\{D_{t_{i}}^{(n)}: n \in M, i \in\right.$ $\left.\left\{1, \ldots, l_{n}\right\}\right\}$;

- if $L_{t_{0}+1}^{\left(n_{0}\right)}=L_{t_{1}}^{\left(n_{0}-1\right)}$ for some $t_{1} \in\left\{0,1, \ldots, 2^{n_{0}-1}-1\right\}$, then at least one of the sets $D_{t_{0}-1}^{\left(n_{0}\right)}, D_{t_{0}}^{\left(n_{0}\right)}, D_{t_{1}-1}^{\left(n_{0}-1\right)}$ belongs to the family $\left\{D_{t_{i}}^{(n)}: n \in M, i \in\right.$ $\left.\left\{1, \ldots, l_{n}\right\}\right\}$;

In each of the above cases, let $D_{\hat{t}}^{(\hat{n})}$ denote this set. Then $\operatorname{proj}_{F}(x) \in F_{\hat{t}}^{(\hat{n})}$ and (by (27)) $g(x) \in f\left(F_{\hat{t}}^{(\hat{n})}\right) \subset \operatorname{cl}\left(F_{\hat{t}}^{(\hat{n})} \cap \Phi\right) \subset \operatorname{cl}(W)$. From the fact that the sets $W$ and $T$ are separated it follows that $g(x) \notin T$. On the other hand, $g(x) \in g(C)=W \cup T$. Hence $g(x) \in W$.

$3^{0}: x=m_{t_{0}+1}^{n_{0}-1\left(n_{0}\right)}$. This case is considered similarly as $2^{0}$.

The inclusion $g(C) \subset W$ has thus been proved. From it and the equality $g(C)=W \cup T$ it follows that $T=\emptyset$, which completes the proof that $g$ is a Darboux function.

Now, we shall demonstrate that $Q_{g}=Q_{f}$. For this purpose, we first prove that $Q_{f} \subset Q_{g}$.Consider $x_{0} \in Q_{f}$. Fix $\varepsilon^{\star}, \delta^{\star}>0$. Then there exist a point $x^{\prime}$ and a number $\delta^{\prime}>0$, such that $\left(x^{\prime}-\delta^{\prime}, x^{\prime}+\delta^{\prime}\right) \subset\left(x_{0}-\delta^{\star}, x_{0}+\delta^{\star}\right)$ and $f\left(\left(x^{\prime}-\delta^{\prime}, x^{\prime}+\delta^{\prime}\right)\right) \subset K\left(f\left(x_{0}\right), \frac{\varepsilon^{\star}}{2}\right)$. Let $n_{0} \in \mathbb{N} \backslash\{0,1\}$ be a positive integer such that $\frac{\varepsilon}{n_{0}}<\frac{\varepsilon^{\star}}{a}$ and such that there are four different half-lines $H_{t}^{\left(n_{0}\right)}$ among those which have occurred in the construction of the function $g_{n_{0}}$ (Denote them $H_{0}, H_{0}^{\star}, H^{0}, H_{\star}^{0}$.), for which

$$
\begin{aligned}
& H_{0} \cap\left(x^{\prime}-\delta^{\prime}, x^{\prime}\right) \neq \emptyset, H^{0} \cap\left(x^{\prime}, x^{\prime}+\delta^{\prime}\right) \neq \emptyset, \\
& H_{0}^{\star} \cap\left(x^{\prime}-\delta^{\prime}, x^{\prime}\right) \neq \emptyset, H_{\star}^{0} \cap\left(x^{\prime}, x^{\prime}+\delta^{\prime}\right) \neq \emptyset
\end{aligned}
$$

and $T_{0} \subset T_{0}^{\star}$ where $T_{0}\left(T_{0}^{\star}\right)$ denotes an open zone between a line which contains $H_{0}$ and a line which contains $H^{0}\left(H_{0}^{\star}\right.$ and $\left.H_{\star}^{0}\right)$. Let $V_{0}=T_{0} \cap \bigcup_{0 \leq \alpha<\frac{1}{n_{0}}} A_{\alpha} \cap$ $K\left(x_{0}, \delta^{\star}\right)$. Clearly, $V_{0}$ is non-empty and open in $\Pi^{+}$, and $V_{0} \subset K\left(x_{0}, \delta^{\star}\right) \cap \Pi^{+}$. 
Note that $g\left(V_{0}\right) \subset K\left(f\left(x_{0}\right), \varepsilon^{\star}\right)$, which proves $\mathbb{Q}_{g} \subset Q_{f}$.

Now, we shall show that $Q_{g} \subset Q_{f}$.Consider $x_{0} \in Q_{g}$. Clearly, $x_{0} \in F$. Fix $\varepsilon^{\star}, \delta^{\star}>0$. Without loss of generality we may assume that $\delta^{\star}<\frac{1}{2}$. Then there exist a point $x^{\prime}$ and a number $\delta^{\prime}>0$, such that $K\left(x^{\prime}, \delta^{\prime}\right) \subset K\left(x_{0}, \delta^{\star}\right)$ and $g\left(K\left(x^{\prime}, \delta^{\prime}\right) \cap \Pi^{+}\right) \subset K\left(f\left(x_{0}\right), \frac{\varepsilon^{\star}}{2}\right)$. Then $K\left(x^{\prime}, \delta^{\prime}\right) \cap \bigcup_{\frac{1}{n_{0}}<\alpha<\frac{1}{n_{0}-1}} A_{\alpha} \neq \emptyset$ for some $n_{0} \geq 2$. Assume that $K\left(x^{\prime}, \delta^{\prime}\right)$ intersects a closed zone between the lines $L_{t_{0}}^{\left(n_{0}\right)}$ and $L_{t_{0}+1}^{\left(n_{0}\right)}$ for some $t_{0} \in\left\{1, \ldots, 2^{n_{0}}-2\right\}$. (If $K\left(x^{\prime}, \delta^{\prime}\right)$ intersects a closed half-plane with edge $L_{1}^{\left(n_{0}\right)}$ which does not contain $L_{2}^{\left(n_{0}\right)}$, or if $K\left(x^{\prime}, \delta^{\prime}\right)$ intersects a closed half-plane with edge $L_{2^{n_{0}}-1}^{\left(n_{0}\right)}$ which does not contain $L_{2^{n_{0}}-2}^{\left(n_{0}\right)}$, the proof is analogous.) Then

$$
K\left(x^{\prime}, \delta^{\prime}\right) \cap P_{t_{0}}^{\left(n_{0}\right)} \cap \bigcup_{\frac{1}{n_{0}}<\alpha<\frac{1}{n_{0}-1}} A_{\alpha} \neq \emptyset
$$

or

$$
K\left(x^{\prime}, \delta^{\prime}\right) \cap\left(P_{t_{0}}^{,\left(n_{0}\right)} \cup P_{t_{0}+1}^{,\left(n_{0}\right)}\right) \cap \bigcup_{\frac{1}{n_{0}}<\alpha<\frac{1}{n_{0}-1}} A_{\alpha} \neq \emptyset .
$$

From (16) and (18) we can conclude that $F_{t_{0}}^{,\left(n_{0}\right)} \cap \Phi \subset g\left(K\left(x^{\prime}, \delta^{\prime}\right) \cap \Pi^{+}\right)$. Then put $W_{0}=\operatorname{proj}_{F}\left(K\left(x^{\prime}, \delta^{\prime}\right)\right) \cap F_{t_{0}}^{\left(n_{0}\right)}$. Clearly, $\operatorname{int}_{F}\left(W_{0}\right) \neq \emptyset$ and $W_{0} \subset$ $\left(x_{0}-\delta^{\star}, x_{0}+\delta^{\star}\right)$. Then

$$
\bigcup_{y \in f\left(W_{0}\right)} K\left(y, \frac{\varepsilon}{n_{0}}\right) \cap \Phi \subset g\left(K\left(x^{\prime}, \delta^{\prime}\right) \cap \Pi^{\star}\right) \subset K\left(f\left(x_{0}\right), \frac{\varepsilon^{\star}}{2}\right) .
$$

Hence

$$
f\left(W_{0}\right) \subset \operatorname{cl}\left(\bigcup_{y \in f\left(W_{0}\right)} K\left(y, \frac{\varepsilon}{n_{0}}\right) \cap \Phi\right) \subset K\left(f\left(x_{0}\right), \varepsilon^{\star}\right),
$$

which proves $Q_{g} \subset Q_{f}$ and consequently $Q_{g}=Q_{f}$.

We shall now show that $C_{g}=C_{f}$. We first prove that $C_{f} \subset C_{g}$. Consider $x_{0} \in C_{f}$. Fix $\varepsilon^{\star}>0$. Let $\delta>0$ be a number such that $f\left(\left(x_{0}-\delta, x_{0}+\delta\right)\right) \subset$ $K\left(f\left(x_{0}\right), \frac{\varepsilon^{\star}}{2}\right)$. Let $n_{0} \in \mathbb{N} \backslash\{0,1\}$ be a positive integer such that $\frac{\varepsilon}{n_{0}}<\frac{\varepsilon^{\star}}{2}$ and such that there are four different half-lines $H_{t}^{\left(n_{0}\right)}$ among those which have occurred in the construction of the function $g_{n_{0}}$ (Denote them $H_{0}, H_{0}^{\star}, H^{0}$, $H_{\star}^{0}$.), for which

$$
\begin{array}{ll}
H_{0} \cap\left(x_{0}-\delta, x_{0}\right) \neq \emptyset, & H^{0} \cap\left(x_{0}, x_{0}+\delta\right) \neq \emptyset, \\
H_{0}^{\star} \cap\left(x_{0}-\delta, x_{0}\right) \neq \emptyset, & H_{\star}^{0} \cap\left(x_{0}, x_{0}+\delta\right) \neq \emptyset
\end{array}
$$


and $T_{0} \subset T_{0}^{\star}$ where $T_{0}\left(T_{0}^{\star}\right)$ denotes an open zone between a line which contains $H_{0}$ and a line which contains $H^{0}\left(H_{0}^{\star}\right.$ and $\left.H_{\star}^{0}\right)$. Let $V_{0}=T_{0} \cap \bigcup_{0 \leq \alpha<\frac{1}{n_{0}}} A_{\alpha} \cap$ $K\left(x_{0}, \delta\right)$. Clearly, $V_{0}$ is a non-empty neighborhood of $x_{0}$ open in $\Pi^{+}$. It is easy to check that $g\left(V_{0}\right) \subset K\left(f\left(x_{0}\right), \varepsilon^{\star}\right)$, which proves $C_{f} \subset C_{g}$. It is also easily seen that $C_{g} \subset C_{f}$, which proves $C_{f}+C_{g}$.

Now, we shall show that $g$ has finite variation.For the purpose, note that

$$
N_{g}(y)= \begin{cases}N_{f}(y), & y \notin \bigcup_{z \in f(F)} K(z, \varepsilon) \cap \Phi, \\ +\infty, & y \in \bigcup_{z \in f(F)} K(z, \varepsilon) \cap \Phi .\end{cases}
$$

Hence $N_{g}(y)=N_{f}(y)$ almost everywhere in $\mathbb{R}^{2}$. Therefore $N_{g}$ is a measurable function, and

$$
\begin{aligned}
& \bigvee_{\mathbb{R}^{2}}(g)=\int_{\mathbb{R}^{2}} N_{g}(y) d y \\
& \left.=\int_{\bigcup_{z \in f(F)} K(z, \varepsilon) \cap \Phi} N_{g}(y) d y+\int_{\mathbb{R}^{2} \backslash\left(\bigcup_{z \in f(F)} K(z, \varepsilon) \cap \Phi\right.}\right) N_{g}(y) d y \\
& \left.=\int_{\mathbb{R}^{2} \backslash\left(\bigcup_{z \in f(F)} K(z, \varepsilon) \cap \Phi\right.}\right) N_{f}(y) d y \leq \int_{\mathbb{R}^{2}} N_{f}(y) d y=\bigvee_{\mathbb{R}^{2}}(f)<+\infty
\end{aligned}
$$

Clearly, $g$ is an $\varepsilon$-extension of $f$. Since $Q_{g}=Q_{f}$ and since $C_{g}=C_{f}$, by (22) $g: \Pi^{+} \rightarrow \mathbb{R}^{2}$ is a Darboux function with finite variation, being an $\varepsilon$-extension of $f$, such that $Q_{g}=Q_{f}$ and $C_{g}=C_{f}$.

Let $S_{F}: \mathbb{R}^{2} \rightarrow \mathbb{R}^{2}$ be a symmetry with respect to the line $F$. We define $f^{\star}: \mathbb{R}^{2} \rightarrow \mathbb{R}^{2}$ by

$$
f^{\star}(x)= \begin{cases}g(x) & \text { if } x \in \Pi^{+}, \\ g\left(S_{F}(x)\right) & \text { if } \left.x \in \mathbb{R}^{2} \backslash \text { int } \Pi^{+}\right) .\end{cases}
$$

It is easy to see that $f^{\star}: \mathbb{R}^{2} \rightarrow \mathbb{R}^{2}$ satisfies the conditions of our theorem.

\section{References}

[1] R. Engelking, Genegal Topology, Warszawa 1977.

[2] St. Lojasiewicz, Wstep do teorii funkcji rzeczywistych, Warszawa 1973.

[3] L. P. Natanson, Theorie der Funktionen einer reelen Veranderlichen, Berlin 1961.

[4] R.J. Pawlak, Variation of Darboux functions, Dem. Math. 22.1 (1989), $115-128$. 
[5] A. Rychlewicz, Funkcje Darboux o wahaniu skonczonym, Rozprawa doktorska.

[6] T. Salat, Generalization of the notion of the Banach indicatrix, Fund. Math. 73 (1971), 29-36.

[7] W. Wilczynski, Superpositions of transformations of bounded variation, Fund. Math. XC, (1976), 211-231. 